\title{
7
}

\section{On Design Evaluation Based on Functional Modeling}

\author{
Stephan Rudolph \\ University of Stuttgart, Institute of Statics and Dynamics of Aerospace \\ Structures, Pfaffenwaldring 27, D-70569 Stuttgart, Germany \\ Telephone: (49) (711) 685-3799, Fax: (49) (711) 685-3706 \\ www: http://www.isd.uni-stuttgart.de/ rudolph \\ email: rudolph@isd.uni-stuttgart.de
}

\begin{abstract}
During early stages of the design process, functional descriptions are often used to describe the design object behavior. Based on the knowledge of such functional descriptions of the design object (i.e. the design parameters contained in these functional relationships), the Pi-theorem is used to derive the associated dimensionless groups. These dimensionless groups are shown to fulfill the necessary conditions commonly expected of evaluation parameters. Based on the validity of the consequently established evaluation hypothesis that "any minimal description in the sense of the Pi-theorem is an evaluation", these automatically generated dimensionless groups then serve as evaluation parameters for the purpose of design object evaluation.

The properties of this evaluation method, such as minimality, completeness, hierarchical decomposition, consistency, causality and sensitivity analysis of the design variables, are derived and discussed. A brief example of the conceptual design of a gas turbine shows the feasibility of the suggested approach. This design evaluation method should in principle be common to the reasoning process of the engineer since it relies on the traditional engineering technique of dimensional analysis.
\end{abstract}

\section{Keywords}

Design evaluation, evaluation hypothesis, functional modeling, dimensional analysis, Pi-theorem.

\section{INTRODUCTION}

Functional modeling means the description of the design object in functional terms. The functional structure of the design object can hereby be described using several functions and their interfaces or flows, see Kuttig (1993). These functions are generally defined as relationships between the input variables, the output variables and the state variables of a system, see Guidelines VDI 2221 (1987). While verbs and nouns are often used for the description of a function and abstract symbols are used for the visualization 
of a function, see Pahl and Beitz (1993), the term 'functional modeling' in this paper is restricted to the description of design objects by functional relationships $f\left(x_{1}, \ldots, x_{n}\right)=0$ of the physical design parameters $x_{1}, \ldots, x_{n}$, such as lengths, Young's modulus or masses.

In all stages of the design process, the design decisions are commonly based on these real design parameters $x_{1}, \ldots, x_{n}$. However, it is shown in this work that these real dimensional quantities do not in general satisfy the necessary conditions for evaluation parameters. It is argued that there exists a strong epistemological reason that only the dimensionless groups $\pi_{j}$ derived from these dimensional quantities $x_{i}$ fulfill the necessary conditions of evaluation parameters.

From design experience, it is also known that there are several levels of difficulty and complexity associated with functional modeling based on design parameters:

- Firstly, the so-called relevance list $x_{1}, \ldots, x_{n}$ of the design parameters involved in the description of the design object function $f\left(x_{1}, \ldots, x_{n}\right)=0$ needs to be established. This relevance list may, however, change during the design process, e.g. when further knowledge is obtained.

- Secondly, the functional relationship $f\left(x_{1}, \ldots, x_{n}\right)=0$ relating the design parameters is often unknown or may change during the design process, e.g. when other solution principles are selected.

The method of dimensional analysis has recently been shown to be able to handle these difficulties, see Rudolph (1995a), since the formalism of dimensional analysis based on the Pi-theorem requires only qualitative information about the relevance list of the physical design parameters $x_{1}, \ldots, x_{n}$ and is ideally suited for processing qualitative physical knowledge encoded in the functional descriptions of the design object. Dimensional analysis has therefore already been applied to engineering design problems in the past, see Dolinskii (1990) and Kloberdanz (1991), where it was used to aid the modeling and helped to gain a deeper understanding of the functional behavior of the design object. In other works dimensional analysis was used as a basis for the technique of qualitative reasoning, see Bhaskar and Nigam (1990). However, these works will not be addressed here any further, since they are the topic of ongoing research, see Rudolph (1995b).

In this work, the notation of dimensional analysis is shown to solve the design evaluation problem, which frequently occurs during design synthesis when choosing among various design alternatives. Section 2 presents the epistemological foundation of the evaluation problem. Section 3 presents the theoretical foundation of the suggested evaluation model. A short application example demonstrating the use of the suggested evaluation method is presented in section 4 . Section 5 closes with a discussion and the conclusions drawn from this work.

\subsection{Evaluation problem}

The overall evaluation of a design object commonly consists of various different partial evaluation aspects, also called evaluation criteria. If one accepts the principle of decomposition of a main criterion (or goal) into several smaller sub-criteria (or subgoals) and thus the aggregation of evaluation components into a global evaluation, respectively, then any evaluation model based on such an assumption can only be a valuable tool for the evaluation of design objects during the design process if at least acceptable answers can be found to the following central questions, see Rudolph (1995a):

- How to structure the used goal criteria hierarchy?

- How to determine the evaluation of various distinct goal criteria?

- How to aggregate multiple goal criteria into one single goal criterion? 


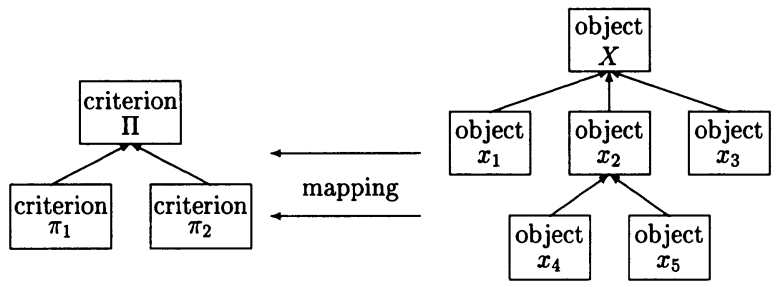

Figure 1 Description, mappings and evaluation

Due to the lack of a formal methodology providing answers to these fundamental questions until now, most classical evaluation models (also called decision making models) require these questions to be answered by a human decision maker, see Hwang and Yoon (1981). The task of the human decision maker is to establish the description graph of the design object, to determine the evaluation graph and then find the corresponding mapping of the description onto the evaluation. This is shown in fig. 1. The influence of the decision maker leads to the central question of to what extent a decision reflects the personal beliefs of the decision maker or whether an objective evaluation should be the unique property of the design object. This is the key idea underlying this work and this issue will be investigated in more detail in the following section.

\section{MOTIVATION}

From a comparative analysis of some existing decision theories and evaluation models in Rudolph (1995a), it can be concluded from epistemological reasoning that

- under the assumption that an objective evaluation exists in general, it may not depend on the arbitrarily chosen definitions of physical units and therefore has to be dimensionless,

- a reproducible and objective evaluation can only exist if it is based on a description derived from some type of law which has to be dimensionally homogeneous,

- an evaluation method should turn into exact physics and be consistent in the case of complete knowledge about a design object.

If the emphasized epistemological requirements mentioned above are considered as necessary conditions of a description in the appropriate implicit mathematical form of a functional relationship $f\left(x_{1}, \ldots, x_{n}\right)=0$ of physical variables, it can be shown that a universal method exists for constructing such dimensionless quantities from dimensionally homogeneous function equations by means of the Pi-theorem. This will be presented in greater detail in the next section.

To ease the understanding of the introduced model, the following terminology will from now on be used as shown in fig. 2 , which is essentially the same diagram as fig. 1 . In fig. $2, x_{i}$ and $X$ represent the description, while $\pi_{j}$ and $\Pi$ represent the corresponding evaluation. The mapping is represented by $\varphi_{0}$ and $\varphi_{j}$, while $\varphi_{1}$ and $\varphi_{3}$ are the respective aggregation functions.

The whole concept of functional modeling is linked to this approach by the fact that $\varphi_{1}$ is the function $f$ relating the physical design parameters $x_{1}, \ldots, x_{n}$. Correspondingly, $\varphi_{3}$ is the function $F$ aggregating the partial evaluations $\pi_{j}$ into a global evaluation. As will be shown later, neither $\varphi_{1}$ nor $\varphi_{3}$ needs to 


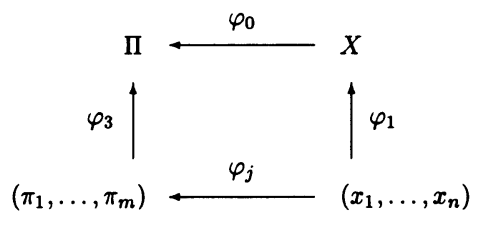

Figure 2 Description, mappings and evaluation

be known explicitly. On the contrary, the knowledge of the dimensional information of the relevance list $x_{1}, \ldots, x_{n}$ of the physical design parameters is sufficient, as shown in the theoretical derivation and the example of the gas turbine. This knowledge is generally available through engineering experience.

\section{FOUNDATION}

The advantageous exploitation of the dimensional analysis in the establishment of a design evaluation model will be shown in the following. In order to do so, the so called Buckingham- or Pi-theorem, see Buckingham (1914) and Bridgman (1922), derived from the theory of dimensionally homogeneous function equations, is shown.

Pi-theorem, Bridgman (1922). From the existence of a dimensionally homogeneous and complete equation $f$ of $n$ physical quantities $x_{i}$ the existence of an equation $F$ of only $m$ dimensionless quantities $\pi_{j}$ can be shown

$$
\begin{aligned}
f\left(x_{1}, \ldots, x_{n}\right) & =0 \\
F\left(\pi_{1}, \ldots, \pi_{m}\right) & =0
\end{aligned}
$$

where $r=n-m$ is the rank of the dimensional matrix constructed by the $x_{i}$ and with dimensionless quantities $\pi_{j}$ of the form

$\pi_{j}=x_{j} \prod_{i=1}^{r} x_{i}^{-\alpha_{j i}}$

with $j=1, \ldots, m \varepsilon \mathbb{N}$ and the $\alpha_{j i} \varepsilon \mathbb{R}$ as constants. (The definition of the dimensional matrix is given in fig. 3 and examples are given in the application section).

The so-called dimensional matrix in the left hand side of fig. 3 has $n$ rows for the design parameters $x_{i}$ and up to $k \leq 7$ columns for the dimensional representation of the parameters expressed in the base units of the employed unit system $(k \leq 7$ is today generally valid in physics, since currently 7 independent physical dimensions, $m_{1} \equiv$ mass, $m_{2} \equiv$ length, $m_{3} \equiv$ time, $m_{4} \equiv$ temperature, $m_{5} \equiv$ current, $m_{6} \equiv$ amount of substance and $m_{7} \equiv$ light intensity in form of the known 7 SI-units $[k g],[m],[s],[K],[A],[m o l]$ and $[c d]$, can be distinguished). 

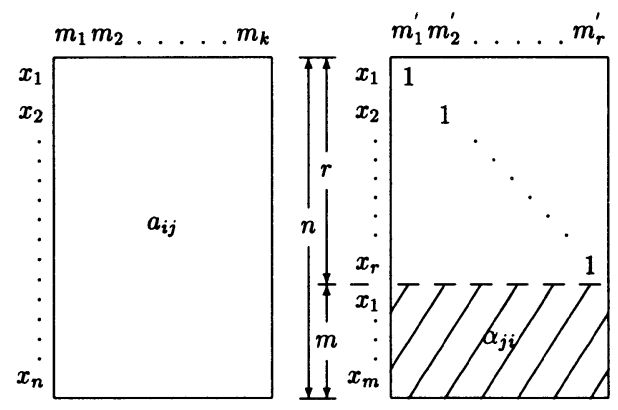

Figure 3 Definition of Dimensional Matrix Computations

To calculate the dimensionless products in equation (2), the dimensional matrix of the design parameters $x_{1}, \ldots, x_{n}$ as shown on the left hand side of fig. 3 needs to be created. Multiples of matrix columns may then be added to each other to obtain the upper diagonal form of the dimensional matrix as shown on the right hand side of fig. 3 . These matrix operations are rank preserving operations in the sense of the linear algebra and therefore lead to a modified dimensional representation of the design variables $x_{i}$ in the original dimensional system $m_{1}, \ldots, m_{k}$ in a new, equivalent dimensional system $m_{1}^{\prime}, \ldots, m_{r}^{\prime}$, with $r \leq k$. The exponents $\alpha_{j i}$ of the dimensionless products in equation (2) are then automatically determined by the values of the coefficients $\alpha_{j i}$ in the hatched part of the matrix on the lower right hand side of fig. 3 . As a consequence of these rank preserving operations on the dimensional matrix, the subset of the design parameters $x_{1}, \ldots, x_{r}$ must be selected from the whole set of design parameters $x_{1}, \ldots, x_{n}$ in such a way that their transformed dimensional representation in the transformed dimensional system results in a diagonal form. This simple procedure is illustrated in the derivation of the dimensionless products of the gas turbine example.

The evaluation hypothesis presented below is used to derive the properties of the evaluation model and can be proved with the help of the Pi-theorem. An evaluation represents a qualitative and quantitative measure of an object or process. As long as its representation form is still redundant, this redundancy can be eliminated without loss of information. Therefore, any evaluation must possess the property of a redundancy-free representation form and has to be minimal in this respect, see Rudolph (1995a). This means that the number of independent evaluation parameters cannot be reduced any further. Since the Pi-theorem exhibits all the required mathematical properties of evaluation parameters, the evaluation hypothesis that "any minimal description in the sense of the Pi-theorem is an evaluation" is postulated.

The application of this evaluation hypothesis as the key idea of the evaluation model is simple. Let $f\left(x_{1}, \ldots, x_{n}\right)=0$ be an arbitrary functional description of the design object, then $F\left(\pi_{1}, \ldots, \pi_{m}\right)=0$ represents the corresponding evaluation. The individual $\pi_{j}$ represent the individual nodes of the evaluation graph in fig. 1, while $F$ represents the aggregation function.

In order to clarify and separate the use of the terms "description parameter" from the term "evaluation parameter" in this paper, it is stressed that a description parameter is considered as a (physical) variable $x_{i}$ possessing a dimensional representation (in the form of the 7 SI-units length in $[m]$, mass in $[k g]$, and so on, or combinations of the seven). Thus, there exists a one-to-one relationship between the design object in reality and its chosen representation form in the form of the design parameters $x_{i}$. The evaluation parameters, however, are the dimensionless groups $\pi_{j}$, which are monomials of the design parameters and 
are therefore a many-to-one relationship between the design object description and its chosen evaluation form in the form of the evaluation parameters. More properties of the evaluation model are presented in the following section.

\section{EVALUATION MODEL}

Using the proof of the Pi-Theorem, the following list of selected properties can be shown for the evaluation method, see Rudolph (1995a):

- Evaluation. The problem of evaluation can be reduced to a problem of description. The problem of evaluation is solved exactly in those cases where a complete description exists.

- Minimality. The dimensionless products form a basis in the sense of a linear vector space. The property of a vector space basis such as minimality is therefore also valid for so-called fundamental systems of dimensionless products.

- Granularity. Adding one more design parameter $x_{n+1}$ to the original description set of $x_{1}, \ldots, x_{n}$ generally adds one more $\pi_{m+1}$ and leaves the original set of evaluation components $\pi_{1}, \ldots, \pi_{m}$ unchanged. This property supports the procedure of hierarchical refinement in the sequence of steps of the design process.

- Hierarchy. Solving $F$ for a specific $\pi_{j}$ immediately creates a consistent hierarchy as shown in fig. 2 . This property can be extended to multiple hierarchies.

- Sensitivity. The differential formulation of the model laws with $\pi_{j}=$ const is $d \pi_{j}=0$. Differentiating equation (2) leads to

$$
d \pi_{j}=\frac{\partial \pi_{j}}{\partial x_{j}} d x_{j}+\sum_{i=1}^{r} \frac{\partial \pi_{j}}{\partial x_{i}} d x_{i} \quad j=1, \ldots, m
$$

and setting $d \pi_{j}=0$ leads to the general form of an iso-line of an evaluation component. If only infinitesimal changes of two design parameters $x_{j}$ and $x_{i}$ are permitted, with all other changes equal to zero, one obtains

$$
\begin{array}{ll}
\frac{\partial x_{j}}{\partial x_{i}}=\alpha_{j i} \frac{x_{j}}{x_{i}} & i=1, \ldots, r \\
& j=1, \ldots, m
\end{array}
$$

which is analogous to the expression derived in Bhaskar and Nigam (1990) for the purpose of "qualitative reasoning".

A few ideas on how the evaluation method can be tested is given by the fulfillment of the following selected epistemological thoughts, see Rudolph (1995a):

- Causality. The evaluation $\Pi$ is determined by the complete description $X$ in the mathematical sense as a necessary and sufficient condition.

- Invariance. The evaluation $\Pi$ is invariant under scale transformations of the physical units employed in the description $X$ of the design object or process.

- Abstraction. Since the same evaluation $\Pi$ is the property of a whole class of similar but well distinct design objects in $X$, the mapping from $X$ to $\Pi$ is mathematically surjective and not injective. 


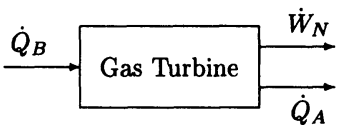

Figure 4 Block Scheme Gas Turbine

Table 1 Dimensional Matrix Calculations Gas Turbine $G$

\begin{tabular}{crrrc}
\hline$G$ & {$\left[m_{1}\right]$} & {$\left[m_{2}\right]$} & {$\left[m_{3}\right]$} & SI units \\
\hline$\dot{Q}_{B}$ & 1 & 2 & -3 & $W$ \\
$\dot{W}_{N}$ & 1 & 2 & -3 & $W$ \\
$\dot{Q}_{A}$ & 1 & 2 & -3 & $W$ \\
\hline
\end{tabular}

- Consistency. Since the evaluation is generated by a mapping, the consistency over multiple hierarchy levels is guaranteed if the theory underlying the design description hierarchy is consistent.

The application of the evaluation method is demonstrated in the next section using the example of the conceptual design of a gas turbine. This example has been chosen because of the fact that analytical solutions are known, thus the outcome of the purely formal dimensional analysis procedure can be compared to this known solution.

\subsection{Gas Turbine Evaluation}

The conceptual design evaluation of a gas turbine based on the functional description of the design parameters is shown. The functional description of the gas turbine is therefore supposed as given, but can be found in most reference books on thermodynamic modeling, such as in Frohn (1977). In a first step, the description of the gas turbine consists of the block scheme in fig. 4. In this way, only those dimensional thermodynamic quantities which cross the system limits need to be considered, see Frohn (1977). These are the thermal power $\dot{Q}_{B}$, chemically supplied in the form of fuel, which is transformed into the (shaft) power $\dot{W}_{N}$ and into (heat) power $\dot{Q}_{A}$. The functional modeling of the gas turbine has in this first step the form $g\left(\dot{Q}_{B}, \dot{W}_{N}, \dot{Q}_{A}\right)=0$. The resulting dimensional matrix $G$ in table 1 with $n=3$ has the rank $r=1$. From the rank preserving operations described in fig. 3 , the two following dimensionless products $\pi_{G 1}$ and $\pi_{G 2}$ are determined according to equation (2) to be equal to

$\pi_{G 1}=\dot{W}_{N} / \dot{Q}_{B}$

$\pi_{G 2}=\dot{Q}_{A} / \dot{Q}_{B}$

From thermodynamic considerations, it follows that $\dot{Q}_{B}$ is partly transformed into $\dot{W}_{N}$ and is partly released as $\dot{Q}_{A}$, resulting in the energy conservation equation $\dot{Q}_{B}=-\dot{W}_{N}-\dot{Q}_{A}$, assuming a systemegotistical position is adopted. Thus, $G\left(\pi_{G 1}, \pi_{G 2}\right)=0$ can be directly determined. However, without this theoretical knowledge, experimental measurements of $\dot{W}_{N}$ and $\dot{Q}_{B}$ are sufficient to obtain the numerical values of the evaluation $\pi_{G 1}$ of the gas turbine efficiency as well and are conform with the classical theoretical findings. Once the the dimensionless product $\pi_{G 1}$ for the evaluation of the power transformation efficiency is obtained, the one-step evaluation hierarchy shown in fig. 5 can be used to represent the current gas turbine evaluation. Additionally, this way of evaluating the gas turbine efficiency using 


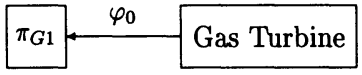

Figure 5 One-step Evaluation Hierarchy

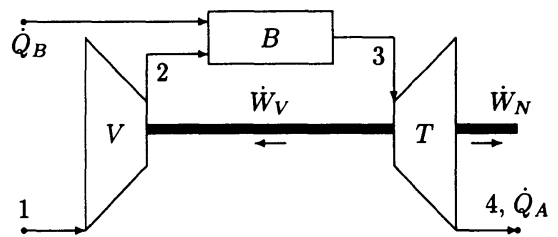

Figure 6 Component Scheme of Gas Turbine

$G\left(\pi_{G 1}, \pi_{G 2}\right)=0$ is consistent with the traditional definition of the thermal efficiency coefficient $\eta_{t h}$, which is defined as

$$
\begin{aligned}
\eta_{t h} & =\frac{-\dot{W}_{N}}{\dot{Q}_{B}}=1+\frac{\dot{Q}_{A}}{\dot{Q}_{B}} \\
& =-\pi_{G 1}=1+\pi_{G 2}
\end{aligned}
$$

Refinement. In the next step of refinement of the functional modeling of the gas turbine, its internal structure with its individual components (compressor $V$, combustion chamber $B$ and turbine $T$ ), is considered in Frohn (1977) in more detail. The resulting modeling is shown in fig. 6. The underlying thermodynamic process is ideally assumed as adiabatic, reversible compression $(1 \rightarrow 2)$, isobaric heating $(2 \rightarrow 3)$, adiabatic-reversible expansion $(3 \rightarrow 4)$ and isobaric cooling $(4 \rightarrow 1)$. The modeling equations stemming from in depth thermodynamic studies of the individual components, see Frohn (1977), are indicated using the appropriate index $V, B$ or $T$.

$$
\begin{aligned}
\dot{W}_{V} & =\dot{m} c_{p} T_{1}\left\{\left(\frac{T_{2}}{T_{1}}\right)-1\right\} \\
\dot{Q}_{B} & =\dot{m} c_{p}\left(T_{3}-T_{2}\right) \\
\dot{W}_{T} & =\dot{m} c_{p} T_{3}\left\{\left(\frac{T_{4}}{T_{3}}\right)-1\right\}
\end{aligned}
$$

However, this exact knowledge is not necessary for the establishment of the evaluation method. For this purpose, only the functional modeling of the three design object components, i.e. of the compressor $V$, the combustion chamber $B$ and the turbine $T$, in the form of the three relevance lists

$$
\begin{array}{r}
f_{V}\left(\dot{m}, c_{p}, T_{1}, \dot{W}_{V}, T_{2}\right)=0 \\
f_{B}\left(\dot{m}, c_{p}, T_{2}, \dot{Q}_{B}, T_{3}\right)=0 \\
f_{T}\left(\dot{m}, c_{p}, T_{3}, \dot{W}_{T}, T_{4}\right)=0
\end{array}
$$


Table 2 Dimensional Matrix Computations

\begin{tabular}{|c|c|c|c|c|c|c|c|c|c|}
\hline$V$ & {$\left[m_{1}\right]$} & {$\left[m_{2}\right]$} & {$\left[m_{3}\right]$} & {$\left[m_{4}\right]$} & SI units & & {$\left[m_{1}\right]$} & {$\left[m_{2}^{\prime}\right]$} & {$\left[m_{3}\right]$} \\
\hline$\dot{m}$ & $\overline{1}$ & & -1 & & $\mathrm{~kg} / \mathrm{s}$ & & 1 & 0 & 0 \\
\hline$c_{p}$ & & 2 & -2 & -1 & $J / k g K$ & & 0 & 1 & 0 \\
\hline$T_{1}$ & & & & 1 & $K$ & $\Rightarrow$ & 0 & 0 & 1 \\
\hline$\dot{W}_{V}$ & 1 & 2 & -3 & & $W$ & & 1 & 1 & 1 \\
\hline$T_{2}$ & & & & 1 & K & & 0 & 0 & 1 \\
\hline$B$ & {$\left[m_{1}\right]$} & {$\left[m_{2}\right]$} & {$\left[m_{3}\right.$} & {$\left[m_{4}\right]$} & SI units & & & & \\
\hline$\dot{m}$ & 1 & & -1 & & $\mathrm{~kg} / \mathrm{s}$ & & 1 & 0 & 0 \\
\hline$c_{p}$ & & 2 & -2 & -1 & $J / k g K$ & & 0 & 1 & 0 \\
\hline$T_{2}$ & & & & 1 & $K$ & $\Rightarrow$ & 0 & 0 & 1 \\
\hline$\dot{Q}_{B}$ & 1 & 2 & -3 & & $W$ & & 1 & 1 & 1 \\
\hline$T_{3}$ & & & & 1 & $K$ & & 0 & 0 & 1 \\
\hline$T$ & {$\left[m_{1}\right]$} & {$\left[m_{2}\right]$} & {$\left[m_{3}\right]$} & {$\left[m_{4}\right]$} & SI units & & & & \\
\hline$\dot{m}$ & 1 & & -1 & & $\mathrm{~kg} / \mathrm{s}$ & & 1 & 0 & 0 \\
\hline$c_{p}$ & & 2 & -2 & -1 & $J / k g K$ & & 0 & 1 & 0 \\
\hline$T_{3}$ & & & & 1 & K & $\Rightarrow$ & 0 & 0 & 1 \\
\hline$\dot{W}_{T}$ & 1 & 2 & -3 & & $W$ & & 1 & 1 & 1 \\
\hline$T_{4}$ & & & & 1 & K & & 0 & 0 & 1 \\
\hline
\end{tabular}

needs to be known. According to these three given modeling equations (12), (13) and (14), three dimensional matrices for compressor $(V)$, the combustion chamber $(B)$ and the turbine $(T)$ can be constructed according to fig. 3. The results are shown in table 2. For each of these three dimensional matrices $V, B$ and $T$ with rank $r=3$ and $n=5$, two dimensionless products are generated by the appropriate rank preserving operations on each dimensional matrix. Once the exponents $\alpha_{j i}$ in the dimensional matrix are determined, the dimensionless products according to equation (2) are found to be equal to

$$
\begin{aligned}
\pi_{V 1} & =\frac{\dot{W}_{V}}{\dot{m} c_{p} T_{1}} \\
\pi_{V 2} & =\frac{T_{2}}{T_{1}} \\
\pi_{B 1} & =\frac{\dot{Q}_{B}}{\dot{m} c_{p} T_{2}} \\
\pi_{B 2} & =\frac{T_{3}}{T_{2}} \\
\pi_{T 1} & =\frac{\dot{W}_{T}}{\dot{m} c_{p} T_{3}} \\
\pi_{T 2} & =\frac{T_{4}}{T_{3}}
\end{aligned}
$$

The resulting evaluation hierarchy is now shown in fig. 7. In this figure, the corresponding abstraction levels of the mapping of the description graph onto the evaluation graph are shown. According to fig. 2, the transformation functions $\varphi_{0}$ and $\varphi_{j}$ coincide with the established dimensionless quantities $\pi_{j}$. The 


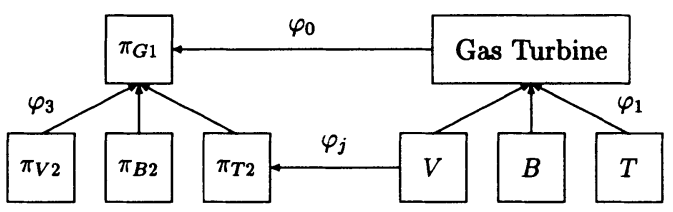

Figure 7 Two-step Evaluation Hierarchy

aggregation function $\varphi_{3}$ of the various partial evaluations into a complete evaluation is not yet determined, since this would require the knowledge of the function $F\left(\pi_{G 1}, \pi_{V 2}, \pi_{B 2}, \pi_{T 2}\right)=0$. This can only be done using theoretical knowledge or experimental data. From the assumption of ideal gases, the global evaluation from the detailed description of the gas turbine is determined by equation (7). By substituting all dimensional quantities into each other, one obtains

$\eta_{t h}=-\pi_{G 1}=1-\frac{\pi_{T 2}-\left(\pi_{V 2} \pi_{B 2}\right)^{-1}}{1-\left(\pi_{B 2}\right)^{-1}}$

It is important to note that the individual nodes of the evaluation graph possess the property of minimality and completeness. Thus, the influence of various changes of the design parameters can now be studied. If an even more detailed description of the functional modeling of the gas turbine is sought (polytropic thermodynamic process modeling, ...), additional dimensionless products for the evaluation model would be obtained. The evaluation model would reflect these additions by the introduction of additional hierarchy levels. The evaluation model hierarchy can thus be extended consistently, see Rudolph (1995a).

\section{DISCUSSION AND CONCLUSION}

The example of the gas turbine evaluation also highlights another source of current misunderstanding and confusion in many engineering design evaluation models concerning the term design description (i.e. the $x_{1}, \ldots, x_{n}$ ) and the term design evaluation (i.e. the $\pi_{1}, \ldots, \pi_{m}$ ). There exist many gas turbines generating different output powers $\dot{W}_{N}$ from different amounts of energy $\dot{Q}_{B}$, but if the numerical value of the (dimensionless) efficiency ratio $\pi_{G 1}=\dot{W}_{N} / \dot{Q}_{B}$ turns out to be the same (i.e. is constant), all these different gas turbines are said to be of the same quality (=efficiency). The output power $\dot{W}_{N}$ alone is therefore clearly a design description parameter, which cannot serve for design evaluation purposes by itself, since its numerical value depends on the arbitrarily chosen physical units (see section 2).

The evaluation method links the problem of design evaluation to the problem of design description. This means that the design evaluation changes exactly in these cases, where the design description is modified. Such modifications may include an

- extension of the relevance list of the design parameters $x_{i}$, which adds one or several lines to the dimensional matrix and finally leads to additional $\pi_{j}$ as stated in section 4 (granularity),

- adaption of every set of $\pi_{1}, \ldots, \pi_{m}$ to the corresponding set of design parameters $x_{1}, \ldots, x_{n}$, since the problem of evaluation is basically reduced to a problem of description, see Rudolph (1995a). 
These features are independent of the explicit knowledge of $f$, since every point set $x_{1}, \ldots, x_{n}$ taken from experiments or gained in simulations implicitly satisfies $f$ and can be mapped without explicit knowledge of $f$ onto the corresponding point set $\pi_{1}, \ldots, \pi_{m}$ in dimensionless space. The method of dimensional analysis can therefore handle many design cases in which the underlying equations and interactions among the design parameters are not (explicitly) known and/or are being discovered during the design process.

\section{CONCLUSION}

By interpretation of the postulated evaluation hypothesis, it is most interesting to note that it can be shown in a rigorous sense, that an evaluation is only possible if a complete description in the form of functional relationships of the design parameters is known. Since complete functional descriptions are generally difficult to obtain, incomplete descriptions in the case of complex design problems will neither allow missing technical aspects to be evaluated, nor give a complete set of dimensionless products. This fact, however, is not a specific weakness of the suggested approach, but reflects the incomplete modeling of the physical phenomena.

Even though the method puts a lot of emphasis on a clear understanding and a complete description of the problem, dimensional analysis based on the Pi-theorem has always been a valuable tool for engineers facing and investigating complex technical problems. Proving the completeness of the relevance list $x_{1}, \ldots, x_{n}$ of parameters is restricted to areas of "sharp" physical knowledge, but the method with its property as a mathematically necessary condition cannot lead to formal contradictions by itself. This makes the method also a versatile tool in areas of "unsharp" physical knowledge. In fact, engineers are usually very good at estimating the relative importance of physical design parameters in all kinds of problems which are theoretically not yet solved. An evaluation method based on the sound establishment of the relevance list $x_{1}, \ldots, x_{n}$ is therefore likely to be applicable in many engineering design cases.

Due to the identical mathematical formulation, the link between classical similarity methods and the new design evaluation methods becomes evident. Such a consistent formulation might ease the application and the understanding of future design systems combining these features. The implementation of the evaluation method into a software tool is described in Rudolph (1995b). In this software module, the manual selection of the design variables $x_{1}, \ldots, x_{n}$ takes the designing engineer very little time. The system then automatically generates the evaluation parameters $\pi_{1}, \ldots, \pi_{m}$ from equation (2) and the sensitivity analysis from equation (4). Once the evaluation module is incorporated into a CAD-application program, it is hoped that the nowadays still manual selection process of the design evaluation parameters can be further automated, despite the fact that modeling seems to be a highly demanding cognitive process even many humans are not good at, see Rudolph (1995b).

The serious investigation of the suggested evaluation method based on functional modeling in more complex applications may therefore be of general interest to the advancement of engineering design and design evaluation in the future.

\section{ACKNOWLEDGMENTS}

The financial support of this work by the Deutsche Forschungsgemeinschaft (DFG) is acknowledged. Thanks go also to Dipl.-Ing. Stefan Essebier for proofreading the 'english' in the paper. 


\section{BIBLIOGRAPHY}

Bhaskar R. and Nigam A. (1990), Qualitative physics using dimensional analysis. Artificial Intelligence 45, 73-111.

Bridgman P. (1922), Dimensional Analysis. Yale University Press, New Haven.

Buckingham, E. (1914), On Physically Similar Systems; Illustration of the Use of Dimensional Equations. Physical Review 4, 345-376.

Dolinskii, I. (1990), Use of dimensional analysis in the construction of mechanical assemblies for optical instruments. Soviet Journal of Optical Technology 57(8), 512-514.

Frohn, A. (1977), Einführung in die technische Thermodynamik. Akademische Verlagsgesellschaft, Wiesbaden.

Guideline VDI 2221 (1987), Systematic Approach to the Design of Systems and Products. (Translation of the German edition 11/1986), VDI-Verlag, Düsseldorf.

Hwang C.-L. and Yoon, K. (1981), Multiple Attribute Decision Making. Lecture Series in Economics and Mathematical Systems, Springer Verlag, Berlin.

Kloberdanz, H. (1991), Rechnerunterstützte Baureihenentwicklung. Fortschrittsberichte Reihe 20, Nummer 40, VDI-Verlag, Düsseldorf.

Kuttig, D. (1993), Potential and limits of functional modelling in the CAD process. Research in Engineering Design 5, 40-48.

Pahl G. and Beitz, W. (1993), Konstruktionslehre. Springer, Berlin. Also available as: Pahl G. and Beitz, W. (1996), Engineering Design. A systematic Approach. Springer Verlag, London.

Rudolph, S. (1995a), Eine Methodik zur systematischen Bewertung von Konstruktionen. Fortschrittsberichte Reihe 1, Nummer 251, VDI-Verlag, Düsseldorf.

Also available in English as: $A$ Methodology for the Systematic Evaluation of Engineering Design Objects. A copy of this translated $\mathrm{Ph} . \mathrm{D}$. thesis is available on request by email from: rudolph@isd.uni-stuttgart.de, or in writing from: ISD Verlag, Stuttgart University, PhD Number 02-94, Pfaffenwaldring 27, D-70569 Stuttgart.

Rudolph, S. (1995b), On a Symbolic CAD-Front-End for Design Evaluation based on the Pi-theorem. In: Preprints of the IFIP WG 5.2 Workshop on Formal Design Methods for Computer Aided Design. Gero, J. and Sudweeks, F. (eds.), Mexico City, June 13-16, 1995, 189-203.

\section{BIOGRAPHY}

Stephan Rudolph is a postdoctoral member of the Institute of Statics and Dynamics of Aerospace Structures (ISD) at Stuttgart University, Germany. His research interests include the application of similarity methods to engineering design and artificial intelligence, namely to design evaluation models, neural networks, genetic algorithms and pattern recognition (see http://www.isd.uni-stuttgart.de/ rudolph).

Besides his aerospace engineering degrees (BSc, MSc and $\mathrm{PhD}$ ) from Stuttgart University he studied abroad at the École Nationale Supérieure d'Ingénieurs des Constructions Aéronautiques (ENSICA) in Toulouse, France, and at the Massachusetts Institute of Technology (MIT) in Cambridge, USA. 